\title{
A partial field decomposition algorithm and its examples for near-field acoustic holography
}

\author{
Kyoung-Uk Nam and Yang-Hann Kim ${ }^{\text {a) }}$ \\ Center for Noise and Vibration Control (NOVIC), Department of Mechanical Engineering, Korea Advanced \\ Institute of Science and Technology (KAIST), Science Town, Daejeon, 305-701, Korea
}

(Received 22 May 2003; revised 12 February 2004; accepted 12 April 2004)

\begin{abstract}
In this paper we introduce a practical algorithm that can accurately implement partial field decomposition for near-field acoustic holography (NAH). Unlike other methods, the algorithm does not require sensors near sources because it uses calculations rather than measured signals in order to decompose a holography image into the images of individual sources. Therefore it makes holography measurement easier than other methods. The algorithm is composed of five steps: measurement of pressure on a hologram plane, estimation of pressure on a source plane, selection of maximum pressure, estimation of the sound field from one source, and estimation of the remaining sound field. In this paper we also report two experiments that verify and demonstrate the algorithm. In one experiment six speakers are operated independently. The other is a vortex shedding experiment. Their results show that the decomposition method is useful for identifying hidden sources and estimating the power of individual sources. (c) 2004 Acoustical Society of America. [DOI: 10.1121/1.1756896]
\end{abstract}

PACS numbers: 43.20.Rq, 43.60.Cg, 43.60.Sx [SPW]

Pages: $172-185$

\section{INTRODUCTION}

The objective of this paper is to introduce a practical algorithm for a partial field decomposition method $^{1}$ of nearfield acoustic holography. ${ }^{2,3}$

Acoustic holography images convey spatial distributions of acoustic pressure, vector plots of intensity, and other acoustic variables. The images can be obtained, not only on a measurement plane but also on any plane of interest. Especially, the image on a source plane is often useful for identifying source characteristics. Figure 1 shows two examples of holography results. Figure 1(a) can be regarded as what can be produced by a monopole. However, it is difficult to interpret Fig. 1(b). In fact, the image of Fig. 1(b) is what is obtained by summing three independent sources (Fig. 2). As shown in Fig. 2, it is our aim to decompose the composite sound image into those from the individual sources.

There have been many attempts to achieve this objective..$^{4-9}$ These methods are based on virtual ${ }^{10}$ or partial coherence, ${ }^{11-13}$ which requires the placement of reference microphones near the sources. That is because those methods use signals obtained by the reference microphones to decompose individual sources, which essentially introduces practical difficulties. One is that prior information on source positions is required before NAH can be applied. It negates one of NAH's valuable properties: It can successfully mimic the spatial characteristics of sound sources. It is also noteworthy that it is often difficult to place reference microphones near the sources, even if the source positions are known.

A decomposition method ${ }^{1}$ that does not require sensors near the sources was recently proposed. The proposed method makes holography measurement easier than other methods because the reference microphones need not be

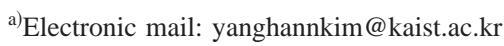

placed near the sources. In spite of this practical advantage, the algorithm of the proposed method was not well addressed: Reference 1 only conveys the theory and basic concepts. In addition, the method's performance, for example, its accuracy, was not verified by experiments.

In this paper we introduce the method's algorithm derived from spectral matrices (instead of the vector notation used in Ref. 1) and also report on two results that were obtained experimentally. The first was a controlled experiment in which six speakers were used to mimic the independent or incoherent sources. In the other, more realistic experiment, it was attempted to separate two sets of vortex shedding noise: noises due to drag and lift forces.

\section{PROBLEM DEFINITION}

In this section we introduce basic equations for the partial field decomposition, referring to Ref. 13. In the following expressions, small letters represent scalar quantities and capital letters express vectors or matrices. All the variables are functions of frequency.

\section{A. Noise rejection in a single-input/single-output system with output noise}

Before introducing the general idea of partial field decomposition, let us consider a single-input/single-output sys-

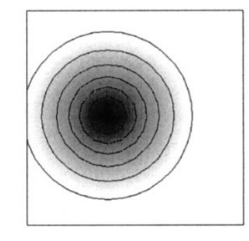

(a) easy to interpret

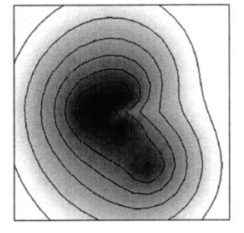

(b) difficult to interpret
FIG. 1. Two examples of holography results, which are pressure distributions at a selected frequency on source planes. 


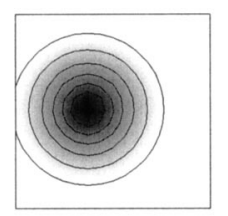

strong monopole

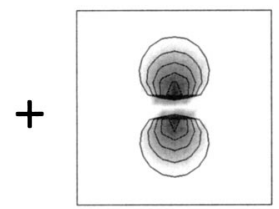

dipole

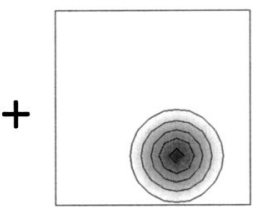

weak monopole

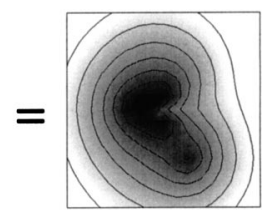

Fig. 1(b)
FIG. 2. Three independent sources of constituting Fig. 1(b). tem with output noise, as shown in Fig. 3(a). The $x$ and $y$ are an input and an output. The $\hat{y}$ and $n$ are measured output and noise. The noise is assumed to be incoherent to the input.

Let $w$ be a signal coherent to the input. That is,

$$
w=c x \text {. }
$$

Then the true output spectrum $s_{y y}$ can be expressed as ${ }^{13}$

$$
s_{y y}=\hat{\gamma}_{w y}^{2} \hat{s}_{y y}=\left|\hat{s}_{w y}\right|^{2} / s_{w w},
$$

where the $\hat{\gamma}_{w y}^{2}$ is the coherence function between the $w$ and $\hat{y}$, the $\hat{s}_{y y}$ is a measured output autospectrum, the $s_{w w}$ is the autospectrum of the $w$, and the $\hat{s}_{w y}$ is the cross-spectrum between the $w$ and $\hat{y}$. Equation (2) means that a true output spectrum can be calculated from a distorted output if a signal coherent to an input is given. ${ }^{13}$ This relation can be readily extended to a two-input/single-output system [Fig. 3(b)].

\section{B. Contribution analysis for a two-input/single-output system}

Let us consider a two-input/single-output system, as shown in Fig. 3(b). The $x_{1}$ and $x_{2}$ are two inputs, the $y_{1}$ and $y_{2}$ are outputs due to the $x_{1}$ and $x_{2}$, and the $y$ is a total output. The two inputs are assumed to be incoherent, considering the circumstance that those are generated by different causes. The total output spectrum $s_{y y}$ can be readily written as $^{13}$

$$
s_{y y}=s_{y 1 y 1}+s_{y 2 y 2},
$$

where the $s_{y l y l}$ is the autospectrum of the $y_{l}$, in other words, the contribution of the $l$ th input to the total output spectrum. In this case, $l=1,2$.

Because the inputs are incoherent to each other, the output due to one input can be regarded as noise to the other input. If a signal $w_{l}$ coherent to the $l$ th input is given, the $l$ th contribution $s_{y l y l}$ can be calculated by ${ }^{13}$

$$
s_{y l y l}=\gamma_{w l y}^{2} s_{y y}=\left|s_{w l y}\right|^{2} / s_{w l w l},
$$

like Eq. (2), where the $\gamma_{w l y}^{2}$ is the coherence function between the $w_{l}$ and $y$, the $s_{w l w l}$ is the autospectrum of the $w_{l}$, and the $s_{w l y}$ is the cross-spectrum between the $w_{l}$ and $y$. Equation (4) essentially expresses what can be applied to a multiple-input/single-output system. ${ }^{13}$ Next, this result is extended to acoustic holography, which has many outputs.

\section{Partial field decomposition in near-field acoustic holography}

Let $N$ be the number of measurement points on a hologram plane and $p_{H n}$ be pressure at the $n$th point. Let $M$ be the number of prediction points on a source plane and $p_{S m}$ be pressure at the $m$ th point. For mathematical convenience, let us define two row vectors as

$$
P_{H}=\left[\begin{array}{lll}
p_{H 1} & \cdots & p_{H N}
\end{array}\right] \text { and } P_{S}=\left[\begin{array}{lll}
p_{S 1} & \cdots & p_{S M}
\end{array}\right] .
$$

These two vectors have the relation of

$$
P_{S}=P_{H} T,
$$

where the $T$ is a matrix of propagating pressure from the hologram to the source plane. ${ }^{2,3}$ The spectral matrices on the hologram and source planes can be defined as

$$
S_{H H}=E\left[P_{H}^{*} P_{H}\right] \text { and } S_{S S}=E\left[P_{S}^{*} P_{S}\right] \text {, }
$$

where the $E$ and * represent expectation and conjugate transpose. Equation (6) rewrites Eq. (7) as

$$
S_{S S}=T^{*} S_{H H} T \text {. }
$$

Let us assume that the number of sources is $L$ and they are incoherent. Then the spectral matrix $S_{S S}$ on the source plane is expressed as

$$
S_{S S}=\sum_{l=1}^{L} S_{S l S l},
$$

which is the matrix version of Eq. (3). The $S_{S l S l}$ expresses how much the $l$ th source contributes to the total spectral matrix $S_{S S}$. In Appendix A we explain the mathematical definition of the $S_{S l S l}$. If $w_{l}$ is a signal coherent to the $l$ th source, the $S_{S l S l}$ can be rewritten as

$$
S_{S l S l}=S_{w l S}^{*} S_{w l S} / s_{w l w l},
$$

where

$$
S_{w l S}=E\left[w_{l}^{*} P_{S}\right],
$$

which is the matrix form of Eq. (4). The $S_{w l S}$ is the row vector representing the cross-spectral matrix between the $w_{l}$

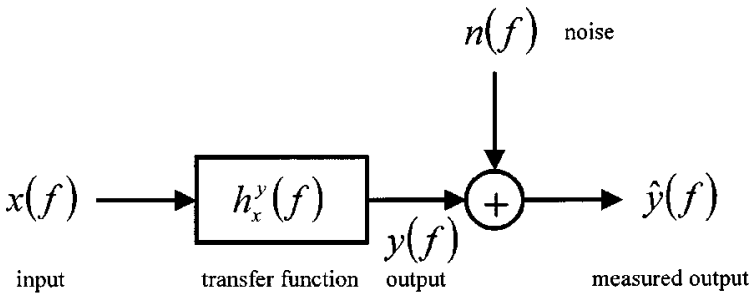

(a) a single-input/single-output system with output noise

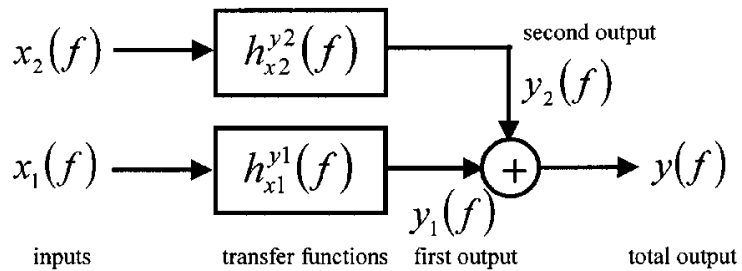

(b) a two-input/single-output system

FIG. 3. Two simple systems for the problem definition of partial field decomposition. 


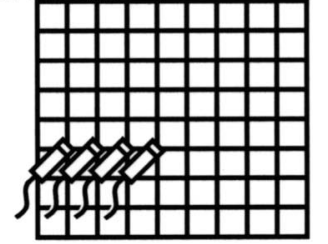

Step 3: Selection of maximum

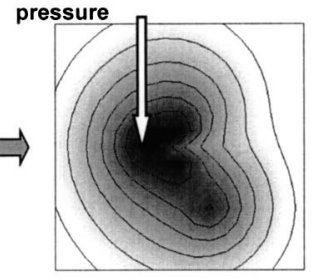

Step 3: Selection of maximum pressure

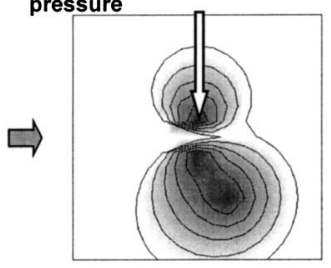

Step 3: Selection of maximum pressure

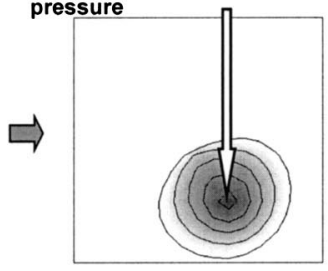

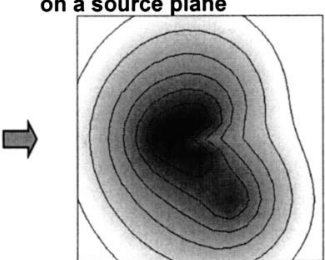

Step 4: Estimation of the

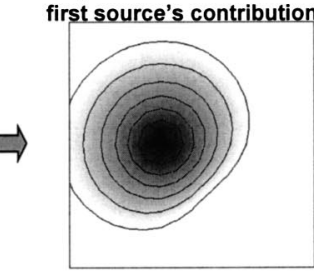

Step 4: Estimation of the second source's contribution

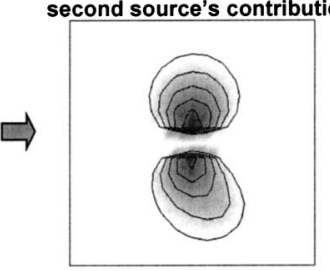

Step 4: Estimation of the third source's contribution

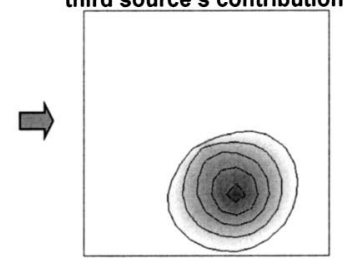

Step 5: Calculation of the remaining field

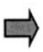

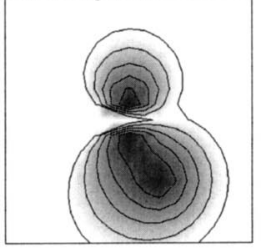

Step 5: Calculation of the remaining field

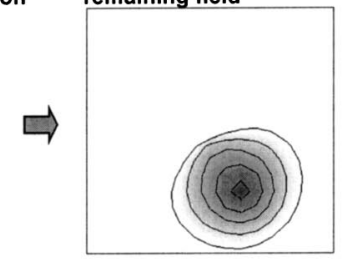

Step 5: Calculation of the remaining field

FIG. 4. An illustration of the decomposition algorithm. This figure illustrates the procedure that separates the sound field in Fig. 1(b). The images of step 4 show the estimated contributions of individual sources.

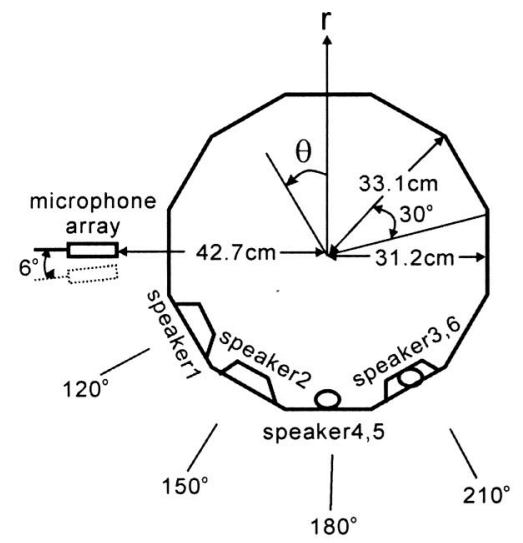

(a) top view

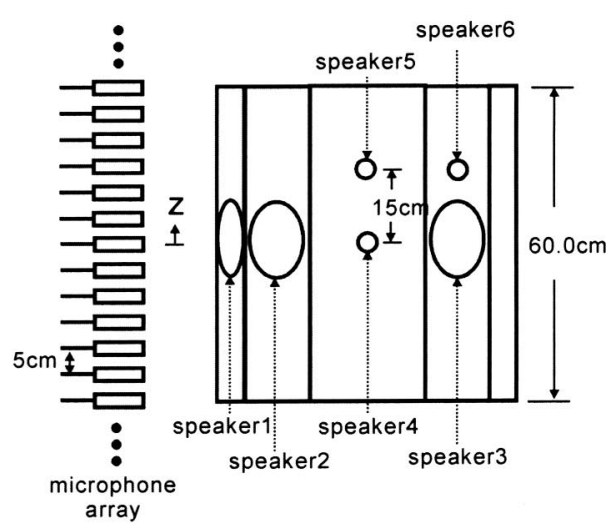

(b) side view
FIG. 5. Setup of the speaker experiment.

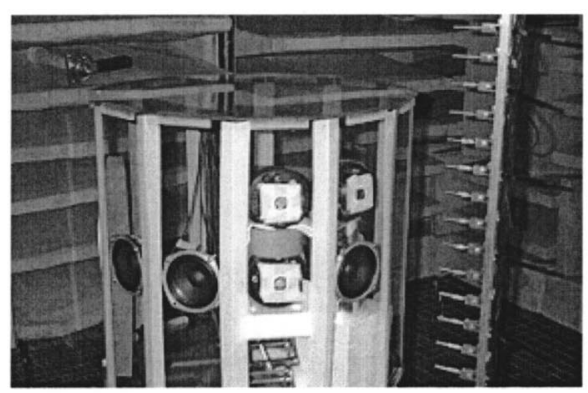

(c) photo 


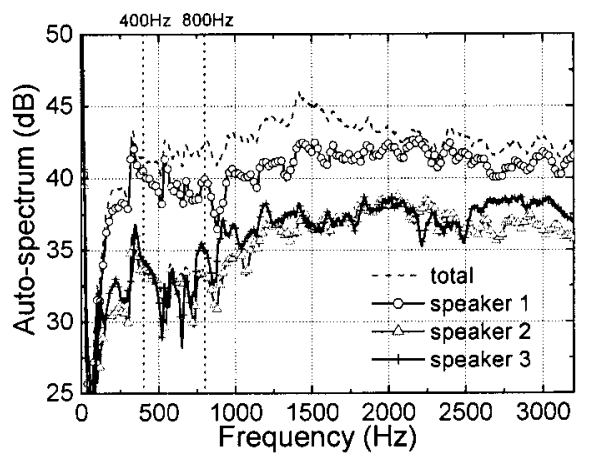

(a) the auto-spectra of speaker $1,2,3$

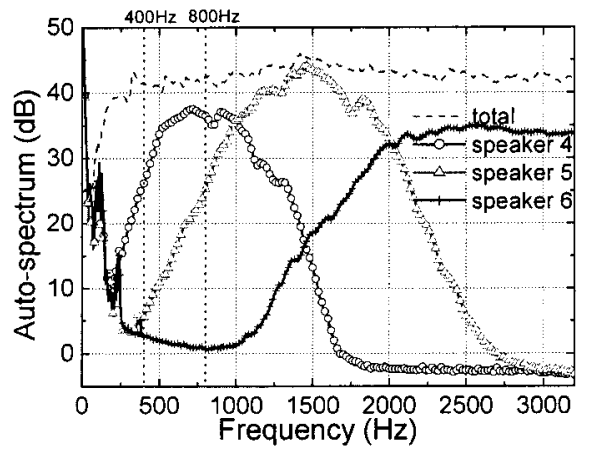

(b) the auto-spectra of speaker $4,5,6$

\begin{tabular}{c|c|c|c|c|c|c}
\hline & speaker1 & 2 & 3 & 4 & 5 & 6 \\
\hline $400 \mathrm{~Hz}$ & $41 \mathrm{~dB}$ & 33 & 34 & 26 & 5 & 2 \\
\hline $800 \mathrm{~Hz}$ & $40 \mathrm{~dB}$ & 33 & 35 & 37 & 25 & 0 \\
\hline
\end{tabular}

(c) the auto-spectra of speakers at 400 and $800 \mathrm{~Hz}$

FIG. 6. Autospectra at one reference microphone in the speaker experiment. Decomposition results will be displayed at 400 and $800 \mathrm{~Hz}$.

and pressure on the source plane. In Appendix B we prove Eq. (10).

This result confirms that one needs signals coherent to individual sources to separate incoherent sources. Conventional methods ${ }^{4-9}$ have obtained such coherent signals by placing sensors near sources.

\section{DECOMPOSITION ALGORITHM}

It is well known that NAH can get a signal coherent to a source signature. Using this rather straightforward idea, Ref. 1 developed a theory, which can effectively separate source shapes. The method assumes that the sound fields from individual sources hardly overlap on the source plane. That is, the method regards maximum pressure on a source plane as a signal coherent to one source. This is essentially equivalent to the idea of placing sensors near the sources. Figure 4 illustrates the details of the procedure that separates the individual noise sources in Fig. 1(b).

The first step measures the hologram spectral matrix $S_{H H}$ [Eq. (7)]. The measurement can be done simultaneously, or by other well established methods. ${ }^{4,5,14,15}$ Let $\hat{S}_{H H}$ be the measured spectral matrix. It is noteworthy that the spectral

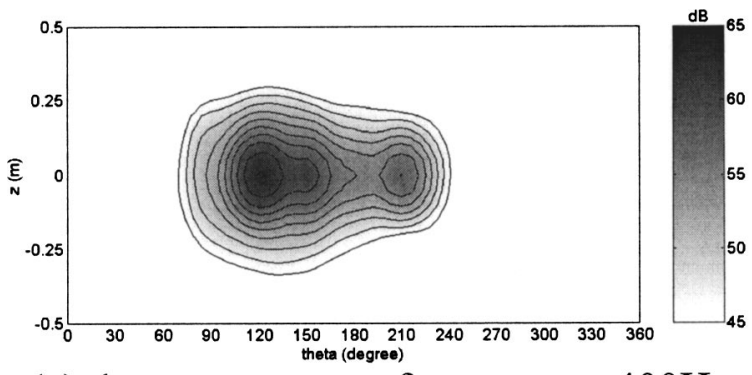

(a) the auto-spectra of pressure at $400 \mathrm{~Hz}$ on the source plane

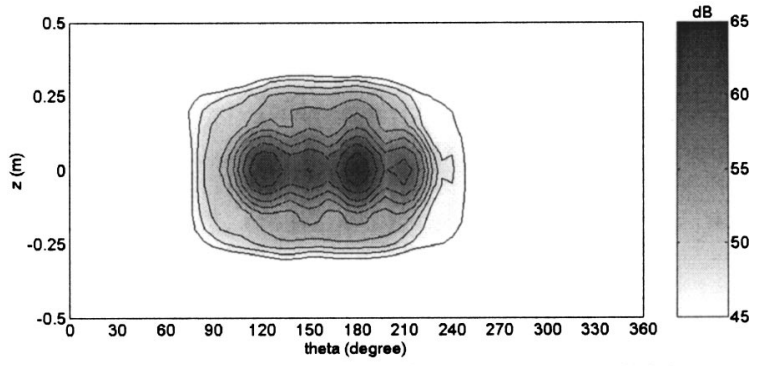

(b) the auto-spectra of pressure at $800 \mathrm{~Hz}$ on the source plane

FIG. 7. Contour plot of the autospectra on the source plane in the speaker experiment.

matrix requires a heavy calculation because of its large size. In order to reduce the calculation time, let us consider a reduced matrix $\hat{\Gamma}_{H}$, which is defined as

$$
\hat{S}_{H H}=\hat{\Gamma}_{H}^{*} \hat{\Gamma}_{H} .
$$

The decomposition of Eq. (12) is possible because $\hat{S}_{H H}$ is Hermitian and semipositive definite. ${ }^{16}$ The $\hat{\Gamma}_{H}$ is a $K \times N$ matrix, where $K$ is the rank of $\hat{S}_{H H}$. The $\hat{\Gamma}_{H}$ is not unique. It can be obtained by singular value decomposition, ${ }^{14} \mathrm{LU}$ decomposition, ${ }^{14}$ or in other ways.

Second, the spectral matrix $S_{S S}$ on the source plane is estimated. Denoting $\hat{S}_{S S}$ as its estimator [Eq. (8)], then it can be written as

$$
\hat{S}_{S S}=T^{*} \hat{S}_{H H} T=T^{*} \hat{\Gamma}_{H}^{*} \hat{\Gamma}_{H} T=\hat{\Gamma}_{S}^{*} \hat{\Gamma}_{S},
$$

where

$$
\hat{\Gamma}_{S}=\hat{\Gamma}_{H} T .
$$

It is noteworthy that the autospectrum at the $m$ th point, the $m$ th diagonal term of $\hat{S}_{S S}$, can be expressed as $\hat{\Gamma}_{S, m}^{*} \hat{\Gamma}_{S, m}$, where $\hat{\Gamma}_{S, m}$ is the $m$ th column of $\hat{\Gamma}_{S}$. The $m$ th row of $\hat{S}_{S S}$ is expressed as $\hat{\Gamma}_{S, m}^{*} \hat{\Gamma}_{S}$. The first and second steps essentially construct the data for acoustic holography.

The third step searches the autospectra on the source plane for maximum pressure. Let the autospectrum $\hat{\Gamma}_{S, m^{\prime}}^{*}, \hat{\Gamma}_{S, m^{\prime}}$ at the $m^{\prime}$ th point be the maximum.

Then, step 4 estimates the contribution of the first source to the spectral matrix by using Eq. (10). By the assumption that maximum pressure is coherent to one source, the $s_{w l w l}$ in Eq. (10) is $\hat{\Gamma}_{S, m}^{*}, \hat{\Gamma}_{S, m^{\prime}}$. The $S_{w l S}$ in Eq. (10) is $\hat{\Gamma}_{S, m}^{*}, \hat{\Gamma}_{S}$. Therefore the estimator $\hat{S}_{S 1 S 1}$ can be written as 


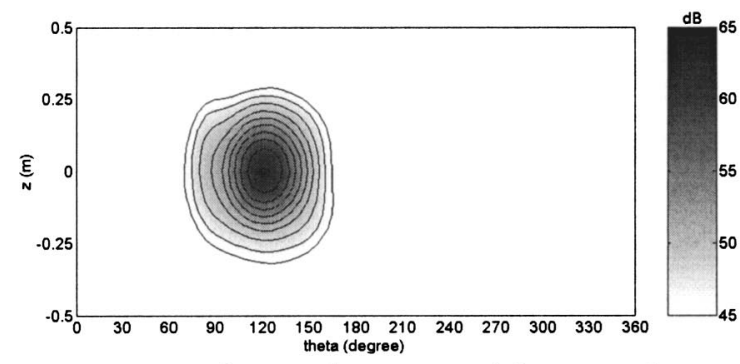

(a) speaker1: decomposition result

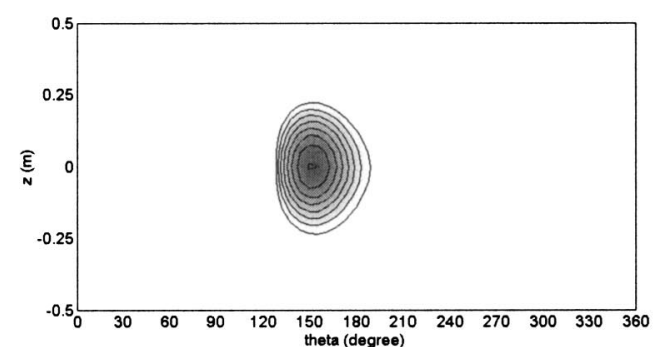

(c) speaker2: decomposition result

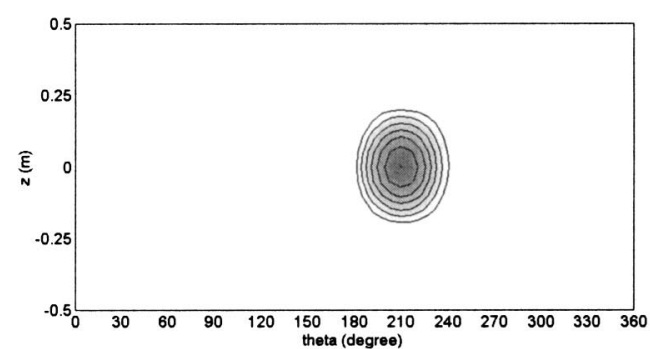

(e) speaker3: decomposition result

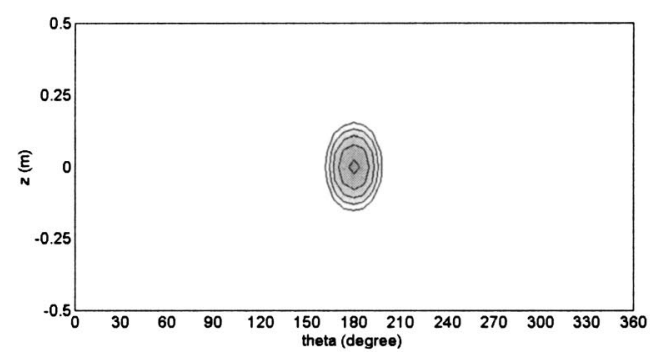

(g) speaker4: decomposition result

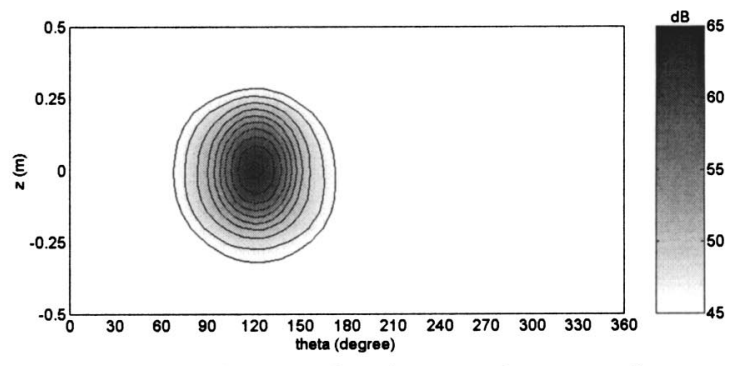

(b) speaker1: single speaker result

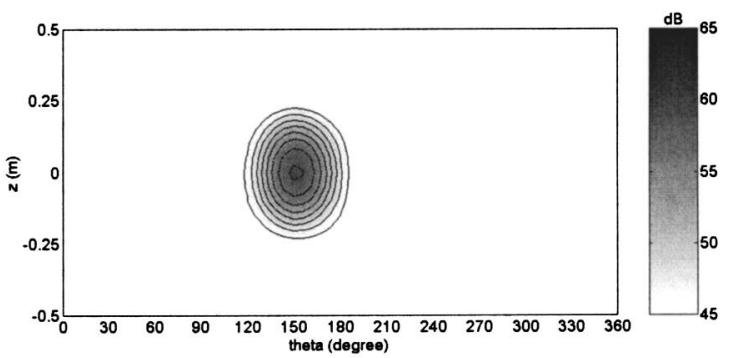

(d) speaker2: single speaker result

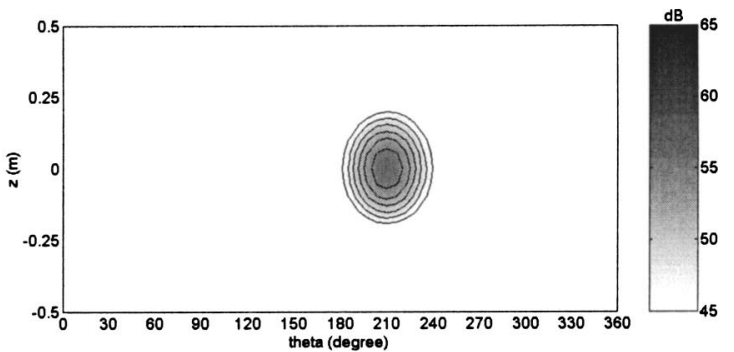

(f) speaker3: single speaker result

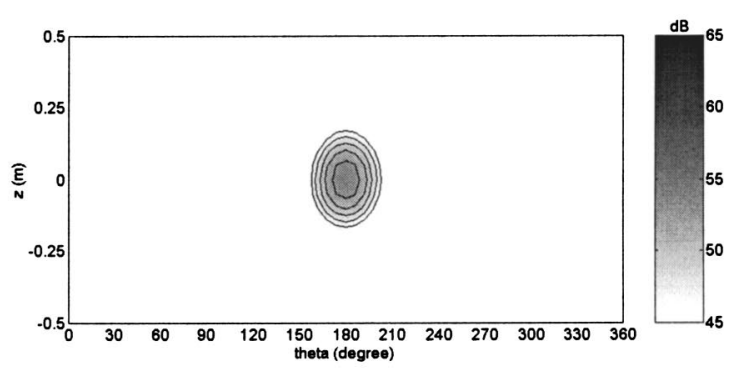

(h) speaker4: single speaker result

FIG. 8. Decomposition results at $400 \mathrm{~Hz}$ in the speaker experiment. The left column shows the decomposition results when all the speakers were operated and the right one exhibits the holography results when only single speaker was operated.

$$
\hat{S}_{S 1 S 1}=\left(\hat{\Gamma}_{S, m^{\prime}}^{*} \hat{\Gamma}_{S}\right) *\left(\hat{\Gamma}_{S, m^{\prime}}^{*}, \hat{\Gamma}_{S}\right) / \hat{\Gamma}_{S, m^{\prime}}^{*} \hat{\Gamma}_{S, m^{\prime}}=\hat{\Gamma}_{S 1^{\prime}}^{*} \hat{\Gamma}_{S 1}
$$

where

$$
\hat{\Gamma}_{S 1}=\hat{\Gamma}_{S, m^{\prime}}^{*} \hat{\Gamma}_{S} / \sqrt{\hat{\Gamma}_{S, m^{\prime}}^{*}, \hat{\Gamma}_{S, m^{\prime}}}
$$

Next (step 5) is to estimate the remaining spectral matrix $S_{S S .1}$, which is the sum of all the source contributions except for the first source. Equations (9), (13), (15), and (16) lead the estimator $\hat{S}_{S S \cdot 1}$ to be expressed as

$$
\hat{S}_{S S \cdot 1}=\hat{S}_{S S}-\hat{S}_{S 1 S 1}=\hat{\Gamma}_{S}^{*} \hat{\Gamma}_{S}-\hat{\Gamma}_{S 1}^{*} \hat{\Gamma}_{S 1}=\hat{\Gamma}_{S \cdot 1}^{*} \hat{\Gamma}_{S \cdot 1},
$$

where

$$
\hat{\Gamma}_{S \cdot 1}=\left(I-\frac{\hat{\Gamma}_{S, m^{\prime}} \hat{\Gamma}_{S, m^{\prime}}^{*}}{\hat{\Gamma}_{S, m^{\prime}}^{*} \hat{\Gamma}_{S, m^{\prime}}}\right) \hat{\Gamma}_{S},
$$

because

$$
\begin{aligned}
& \hat{\Gamma}_{S}^{*}\left(I-\frac{\hat{\Gamma}_{S, m^{\prime}} \hat{\Gamma}_{S, m^{\prime}}^{*}}{\hat{\Gamma}_{S, m^{\prime}}^{*} \hat{\Gamma}_{S, m^{\prime}}}\right)^{*}\left(I-\frac{\hat{\Gamma}_{S, m^{\prime}} \hat{\Gamma}_{S, m^{\prime}}^{*}}{\hat{\Gamma}_{S, m^{\prime}}^{*} \hat{\Gamma}_{S, m^{\prime}}}\right) \hat{\Gamma}_{S} \\
& \quad=\hat{\Gamma}_{S}^{*}\left(I-\frac{\hat{\Gamma}_{S, m^{\prime}}, \hat{\Gamma}_{S, m^{\prime}}^{*}}{\hat{\Gamma}_{S, m^{\prime}}^{*}, \hat{\Gamma}_{S, m^{\prime}}}\right) \hat{\Gamma}_{S}=\hat{\Gamma}_{S}^{*} \hat{\Gamma}_{S}-\hat{\Gamma}_{S 1}^{*} \hat{\Gamma}_{S 1} .
\end{aligned}
$$

This fifth step basically follows a partial coherence procedure. $^{11-13}$ 


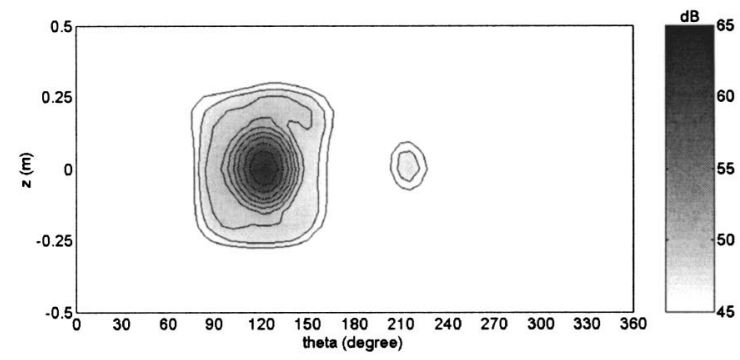

(a) speaker1: decomposition result

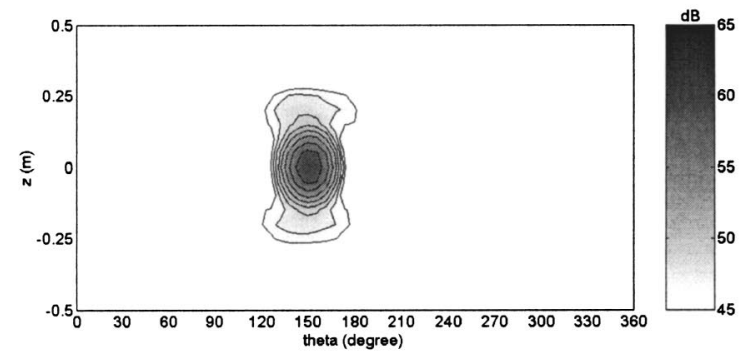

(c) speaker2: decomposition result

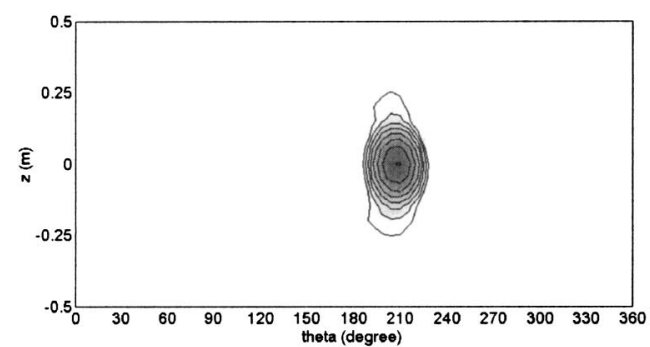

(e) speaker3: decomposition result

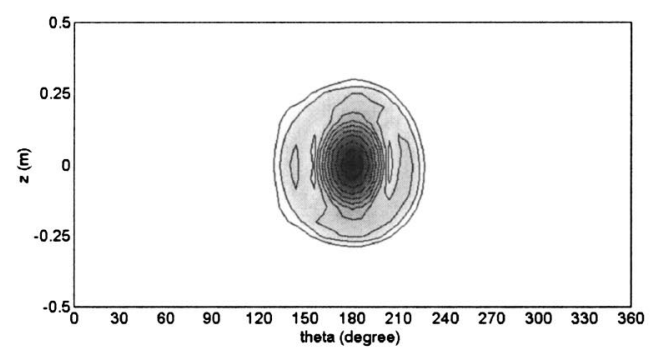

(g) speaker4: decomposition result

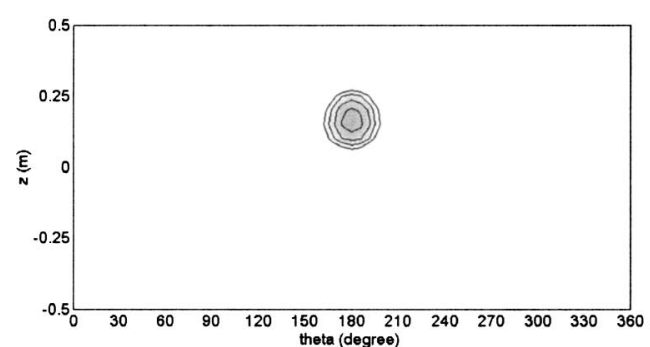

(i) speaker5: decomposition result

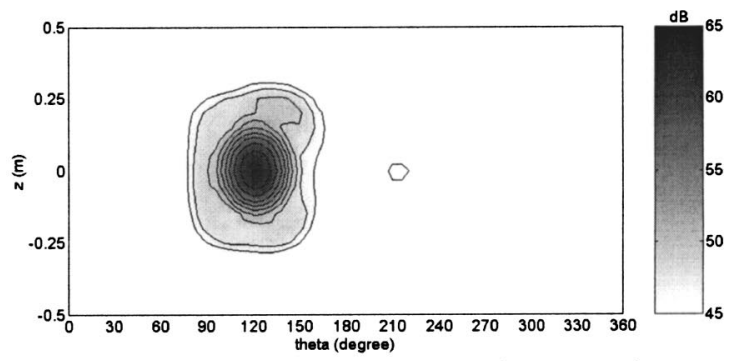

(b) speaker1: single speaker result

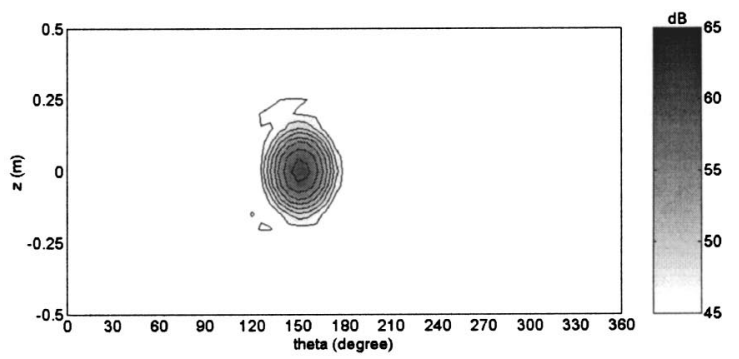

(d) speaker2: single speaker result

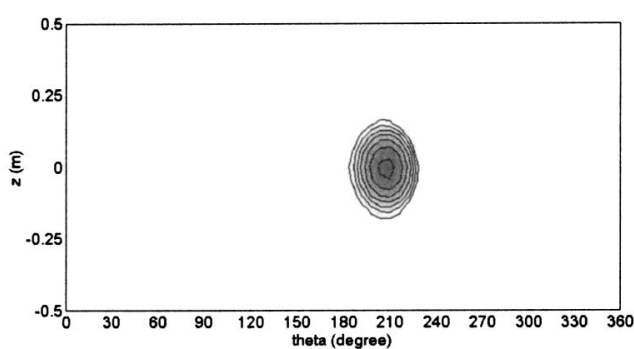

(f) speaker3: single speaker result

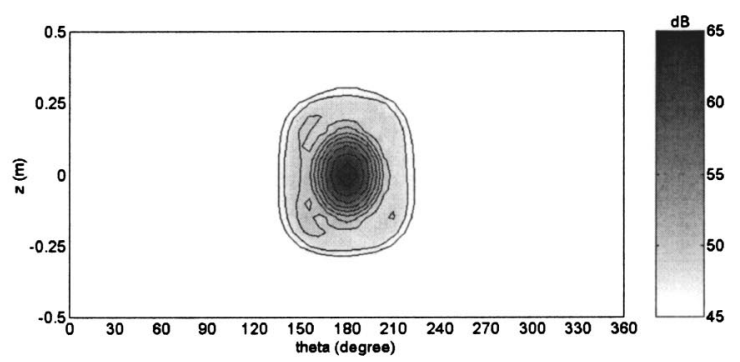

(h) speaker4: single speaker result
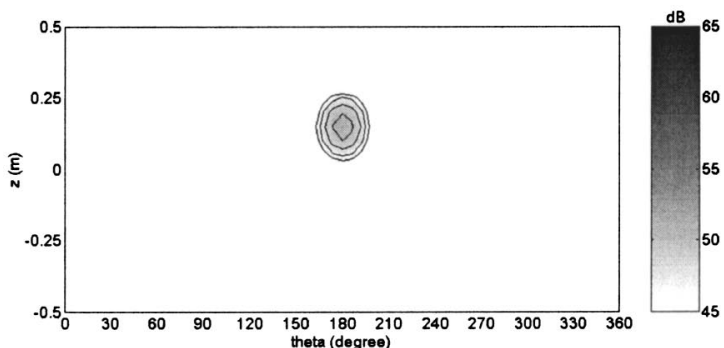

(j) speaker5: single speaker result

FIG. 9. Decomposition results at $800 \mathrm{~Hz}$ in the speaker experiment. The left column shows the decomposition results when all the speakers were operated and the right one exhibits the holography results when only single speaker was operated.

One has to repetitively apply steps $3-5$ to the remaining spectral matrix $\hat{S}_{S S \cdot 1}$ to estimate the contributions of other sources. The maximum number of repetitions is the rank $K$ of the $\hat{S}_{H H}$ because the remaining spectral matrix is zero after the Kth repetition.
The results of Fig. 4 are a little distorted in comparison with Fig. 2. That is because individual sound fields are partially overlapped. The spatial overlap makes the pressure at the maximum point not perfectly induced by one source. Therefore the quality of the proposed method depends on 


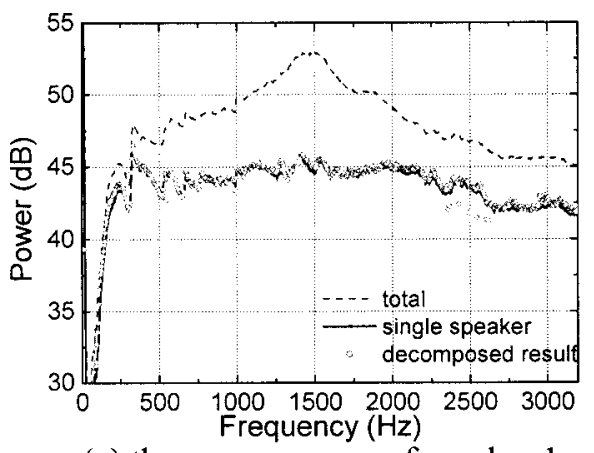

(a) the source power of speaker 1

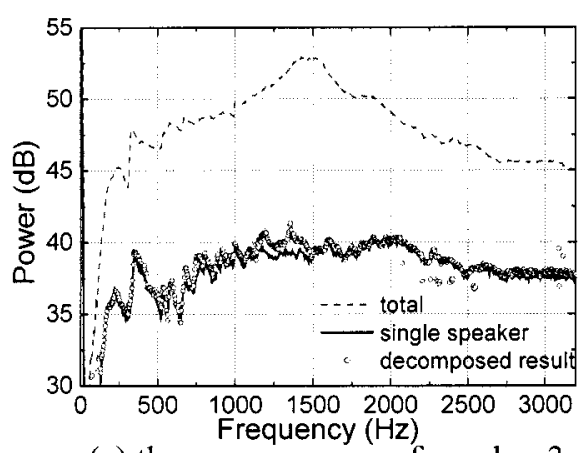

(c) the source power of speaker 3

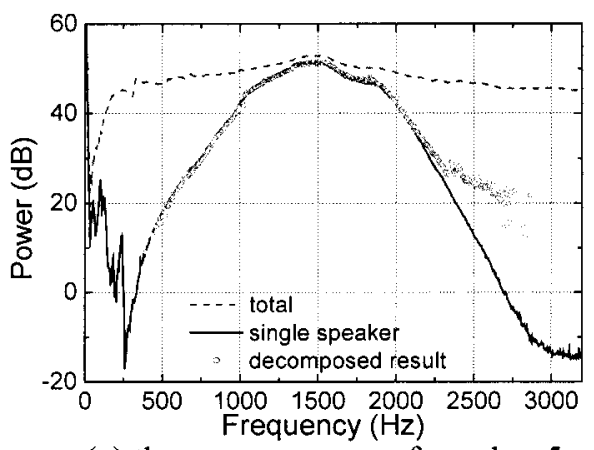

(e) the source power of speaker 5

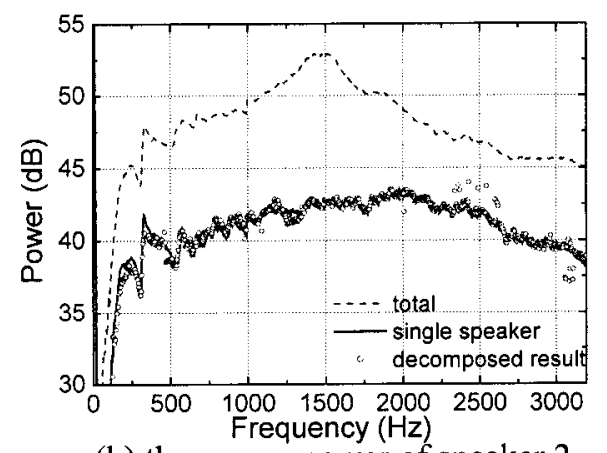

(b) the source power of speaker 2

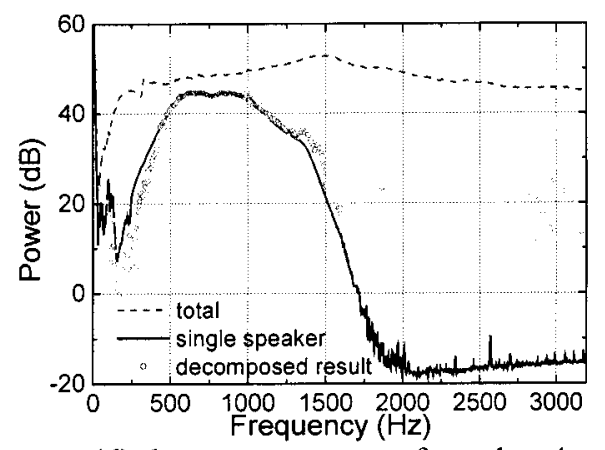

(d) the source power of speaker 4

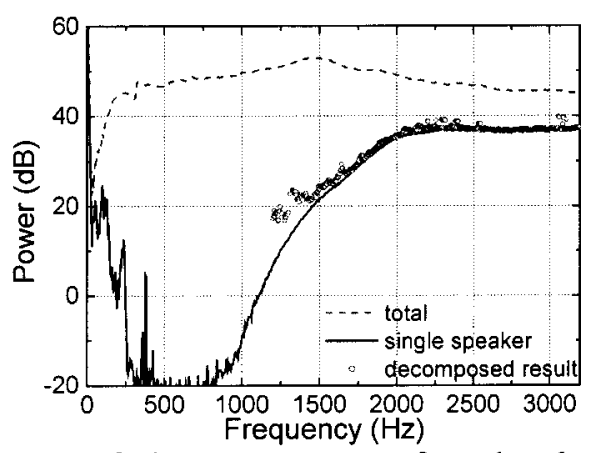

(f) the source power of speaker 6
FIG. 10. Decomposition results of the source power in the speaker experiment. how strongly the sound fields, generated by individual sources, overlap one another. In Appendix $\mathrm{C}$ we introduce some examples of the decomposition error due to the spatial overlap.

In the next two sections we present results from two experiments that demonstrate, as well as verify, the algorithm.

\section{SIX-SPEAKER EXPERIMENT}

Figure 5 illustrates the experimental setup with six sound sources. Speakers 1, 2, 3 are loud speakers of 4 in. diameters and speakers 4, 5, 6 are horn drivers. The speakers were fixed on the wall of an acryl box of $0.5 \mathrm{~cm}$ thickness. Six function generators independently operated the speakers. A cylindrical holography was performed. The radii of the hologram and source planes were 42.7 and $33.1 \mathrm{~cm}$. The measurement spacings in the $\theta$ and $z$ direction were $6^{\circ}$ and 5 $\mathrm{cm}$. The numbers of measurement points in the $\theta$ and $z$ direction were 60 and 24 . For the construction of the hologram spectral matrix $\hat{S}_{H H}$ [Eq. (7)], a 24-microphone array scanned the hologram points step-by-step in the $\theta$-direction.
Six reference microphones were used and located about $1 \mathrm{~m}$ away from the source plane. In Appendix D we provide details on some issues related to this step-by-step scanning method.

The maximum frequency and frequency resolution were 3200 and $4 \mathrm{~Hz}$, respectively. For the spectra calculation, a Hanning window was used and the time data overlapped by $50 \%$. The average number was 40 . For the estimation of pressure on the source plane, the spatial window minimizing errors $^{17}$ and the wave number filter proposed by Veronesi and Maynard $^{18}$ were used. Zeros were added outside the measurement area so that the number of data including the zeros in the $z$ direction was 64 .

For the comparison of the decomposition results, the data from each single speaker were also measured. Figure 6 shows the autospectra of pressure at one reference microphone. Speakers 1, 2, and 3 generated nearly white noise while speakers 4,5 , and 6 generated bandlimited noise. Their main bands were 500-1000, 1000-2000, and 2000-3200 $\mathrm{Hz}$.

Figure 7 shows the autospectra of the pressure at 400 and $800 \mathrm{~Hz}$ on the source plane. As shown in Fig. 6, the 


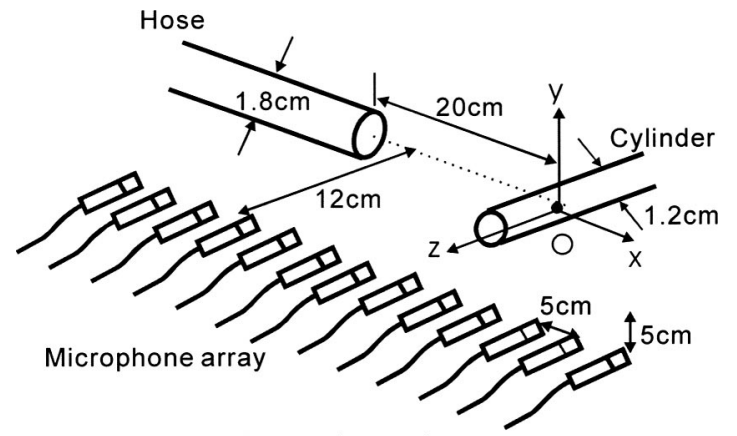

(a) experimental setup

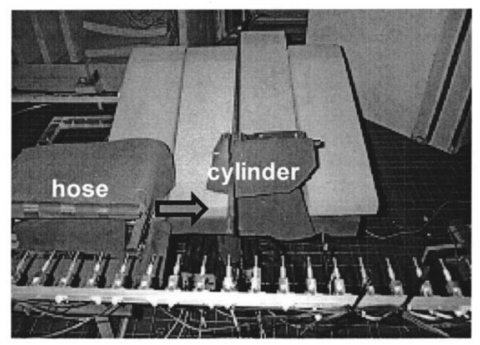

(b) photo

FIG. 11. Setup of the vortex shedding experiment.

noise was mainly generated by speakers $1,2,3$, and 4 at 400 $\mathrm{Hz}$ and by speakers 1, 2, 3, 4, and 5 at $800 \mathrm{~Hz}$. However, all the sources are not clearly seen in Fig. 7.

Figure 8 shows the decomposition results at $400 \mathrm{~Hz}$. The left column shows the decomposition results and the right one exhibits the holography results of the single speaker experiments. The decomposition results clearly demonstrate that the sound fields from speakers 1-4 were well decomposed. The result from the speaker 2 is a little distorted near the peak point of speaker 1 (Appendix C). Because the number of reference microphones is six, the rank of the spectral matrix is also six. ${ }^{4}$ Therefore the number of the estimated contributions is six. At $400 \mathrm{~Hz}$, however, the fifth and sixth contributions were so small that they were not seen in the color range of Fig. 8. Figure 9 shows the decomposition results at $800 \mathrm{~Hz}$. The hidden source, speaker 5, is also seen. The distortions of the shapes at $800 \mathrm{~Hz}$ are smaller than those at $400 \mathrm{~Hz}$ because the spatial overlap is smaller at the higher frequency.

Figure 10 shows the decomposition results of the source power. The results from speakers 1,2 , and 3 were very simi-

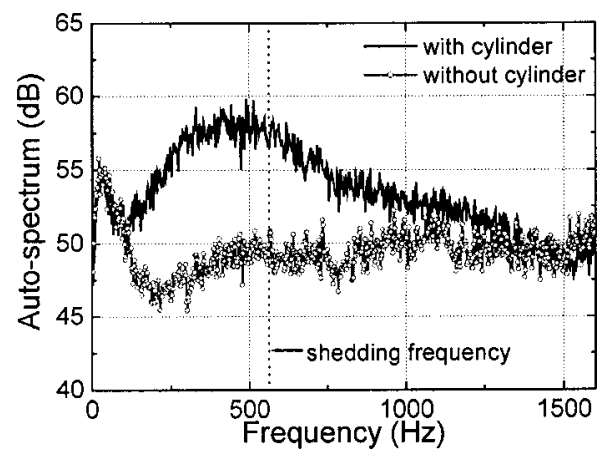

FIG. 12. Autospectra at $(0,15 \mathrm{~cm})$ on the hologram plane in the vortex shedding experiment.

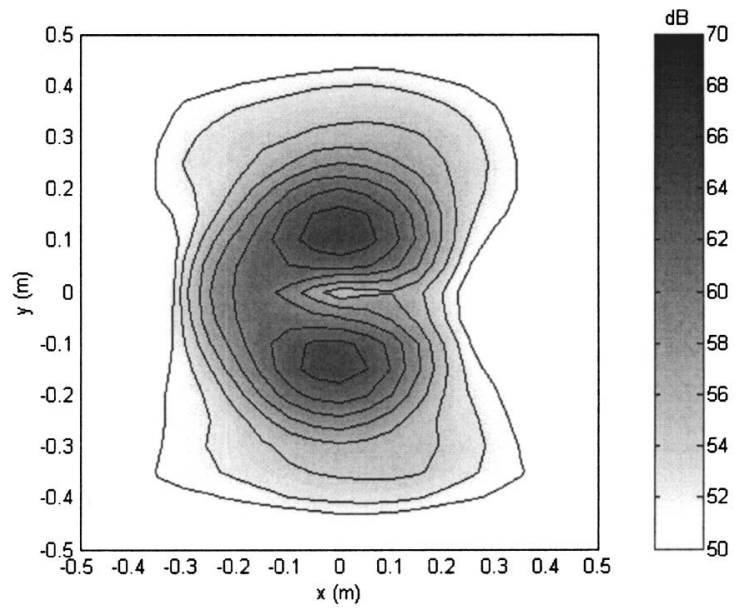

(a) the auto-spectra of pressure at $500 \mathrm{~Hz}$ on the source plane

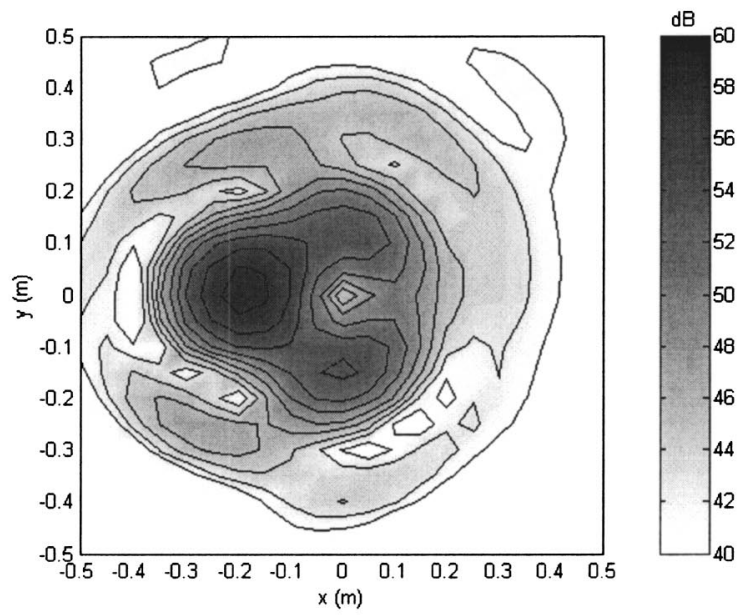

\section{(b) the auto-spectra of pressure at $1000 \mathrm{~Hz}$ on the source plane}

FIG. 13. Contour plot of autospectra on the source plane in the vortex shedding experiment.

lar to the single speaker results at almost all the frequencies. The results from speakers 4,5 , and 6 were also very similar to the single speaker results in their main bands. However, the results had large errors at the frequencies with low-power levels, as discussed in Appendix C. Especially, speakers 4, 5, and 6 at the frequency bands of very low levels, for example, $1500-3200 \mathrm{~Hz}$ of Fig. 10(d), were rarely observed.

\section{VORTEX SHEDDING EXPERIMENT}

Figure 11 shows the experimental setup that generates a vortex shedding. Compressed air was injected into a circular cylinder from a hose. The positions of the hose end and cylinder were $(-20 \mathrm{~cm}, 0)$ and $(0,0)$ in the $x-y$ plane. The diameters of the hose and cylinder were 1.8 and $1.2 \mathrm{~cm}$. The mean velocity of the flow was $33.8 \mathrm{~m} / \mathrm{s}$ at the origin where the flow meets the cylinder. Planar holography was used to visualize the sound field. The distance between the hologram and source plane was $12 \mathrm{~cm}$. The measurement spacing was $5 \mathrm{~cm}$. The number of measurement points was $24 \times 24$. A 


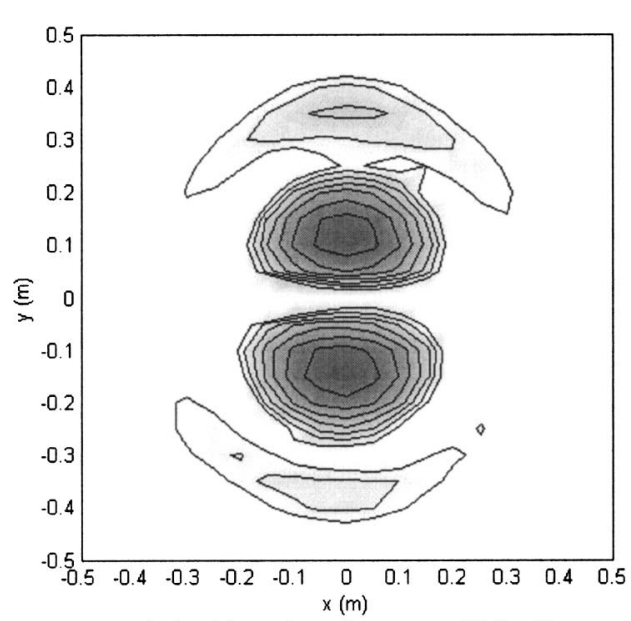

(a) dipole due to lift force

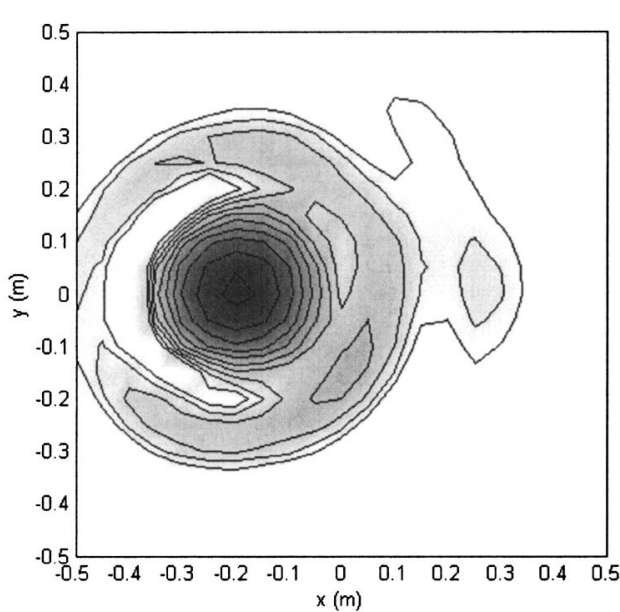

(c) hose end noise
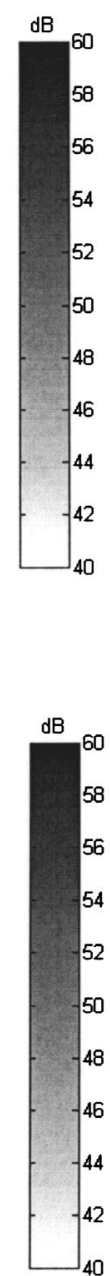

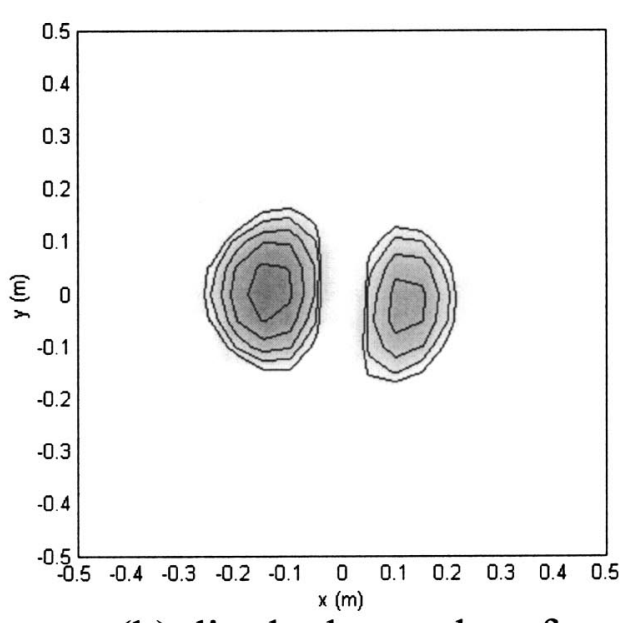

(b) dipole due to drag force
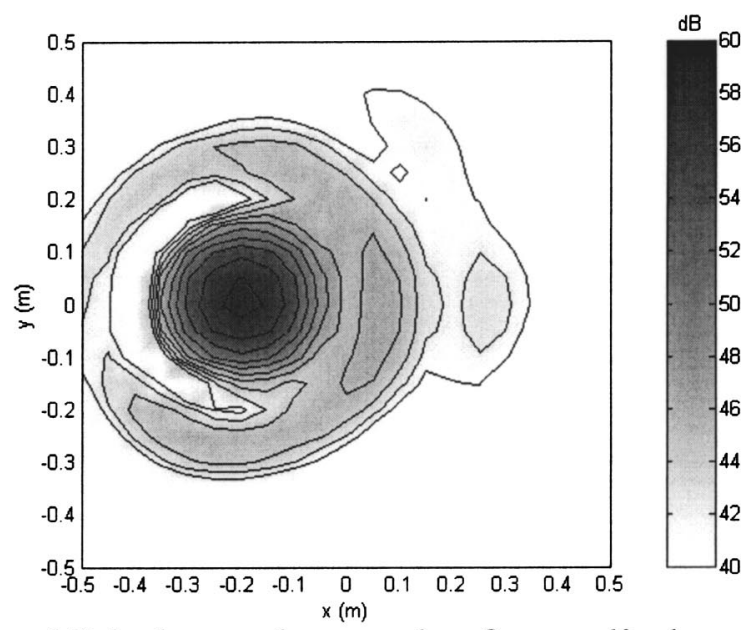

(d) holography result of no cylinder

FIG. 15. Decomposition results at $1000 \mathrm{~Hz}$ in the vortex shedding experiment.

this paper we also reported two experiments that verify the algorithm and certainly demonstrate how it is useful. The experimental results showed that it is useful for identifying hidden sources and estimating the power of individual sources.

\section{ACKNOWLEDGMENTS}

This study was partly supported by the NRL (National Research Laboratory) project of KISTEP (Korea Institute of Science \& Technology Evaluation and Planning), the BK21 (Brain Korea 21) project initiated by the Ministry of Education \& Human Resources \& Development of Korea, and the Hyundai Motor Company \& Kia Motor Corporation.

\section{APPENDIX A: MATHEMATICAL DEFINITION OF $\boldsymbol{s}_{S I S I}$ IN EQ. (9)}

Let $h_{x l}^{S m}$ be the transfer function between the $l$ th source input $x_{l}$ and the pressure at the $m$ th point on the source plane. Let us define a row vector as

$$
H_{x l}^{S}=\left[\begin{array}{lll}
h_{x l}^{S 1} & \cdots & h_{x l}^{S M}
\end{array}\right] .
$$

If the inputs are incoherent and $s_{x l x l}$ is the autospectrum of $x_{l}$, the spectral matrix $S_{S S}$ in Eq. (7) can be expressed as ${ }^{11}$

$$
\begin{aligned}
S_{S S} & =\left[\begin{array}{c}
H_{x l}^{S} \\
\vdots \\
H_{x L}^{S}
\end{array}\right] *\left[\begin{array}{lll}
S_{x l x l} & & \\
& \ddots & \\
& & S_{x L x L}
\end{array}\right]\left[\begin{array}{c}
H_{x l}^{S} \\
\vdots \\
H_{x L}^{S}
\end{array}\right] \\
& =\sum_{l=1}^{L} s_{x l x l} H_{x l}^{S^{*}} H_{x l}^{S} .
\end{aligned}
$$

Therefore $S_{S l S l}$ in Eq. (9) is $s_{x l x l} H_{x l}^{S^{*}} H_{x l}^{S}$.

\section{APPENDIX B: PROOF OF EQ. (10)}

Let $w_{l}$ be coherent to the lth source input $x_{l}$ as

$$
w_{l}=c_{l} x_{l} \text {. }
$$

Let $s_{x l S m}$ be the cross-spectrum between the $x_{l}$ and $p_{S m}$, and $s_{w l S m}$ be the cross-spectrum between the $w_{l}$ and $p_{S m}$. Then the $S_{w l S}$ in Eq. (11) can be rewritten as

$$
S_{w l S}=\left[\begin{array}{lll}
s_{w l S 1} & \cdots & s_{w l S M}
\end{array}\right]=c_{l}^{*}\left[\begin{array}{lll}
s_{x l S 1} & \cdots & s_{x l S M}
\end{array}\right] .
$$

Because the inputs are incoherent, the $h_{x l}^{S m}$ in Appendix A is expressed as ${ }^{13}$

$$
h_{x l}^{S m}=s_{x l S m} / s_{x l x l} \text {. }
$$




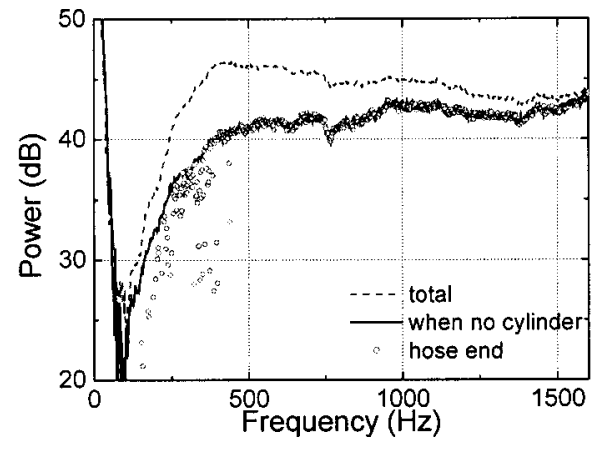

(a) the source power of hose end noise

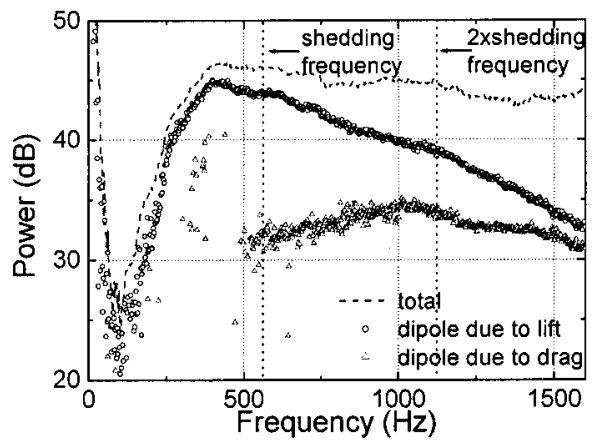

(b) the source power of dipoles

FIG. 16. Decomposition results of source power in the vortex shedding experiment.

Therefore Eqs. (A1) and (B1)-(B3) derive Eq. (10) as

$$
\begin{aligned}
S_{w l S}^{*} S_{w l S} / s_{w l w l}= & c_{l}^{*}\left[\begin{array}{lll}
s_{x l S 1} & \cdots & s_{x l S M}
\end{array}\right]^{*} \\
& \times c_{l}\left[\begin{array}{lll}
s_{x l S 1} & \cdots & s_{x l S M}
\end{array}\right] /\left|c_{l}\right|^{2} s_{x l x l} \\
= & s_{x l x l}\left[\begin{array}{llll}
s_{x l S 1} / s_{x l x l} & \cdots & s_{x l S M} / s_{x l x l}
\end{array}\right]^{*} \\
& \times\left[\begin{array}{llll}
s_{x l S 1} / s_{x l x l} & \cdots & s_{x l S M} / s_{x l x l}
\end{array}\right] \\
= & s_{x l x l}\left[\begin{array}{llll}
h_{x l}^{S 1} & \cdots & h_{x l}^{S M}
\end{array}\right]^{*}\left[\begin{array}{llll}
h_{x l}^{S 1} & \cdots & h_{x l}^{S M}
\end{array}\right] \\
= & s_{x l x l} H_{x l}^{S *} H_{x l}^{S}=S_{S l S l} .
\end{aligned}
$$

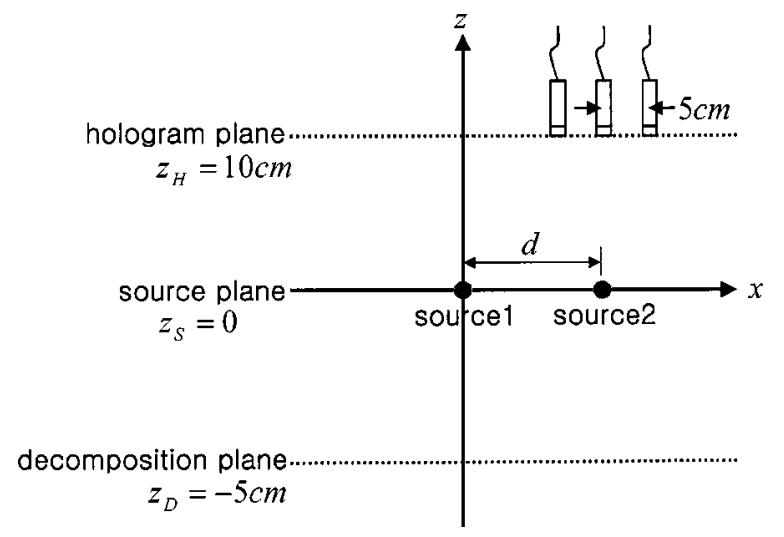

FIG. 17. Specifications for the numerical examples of a decomposition error. Variables are source types (monopole or dipole), distance $d$ between the two sources, and source strength (or peak level).

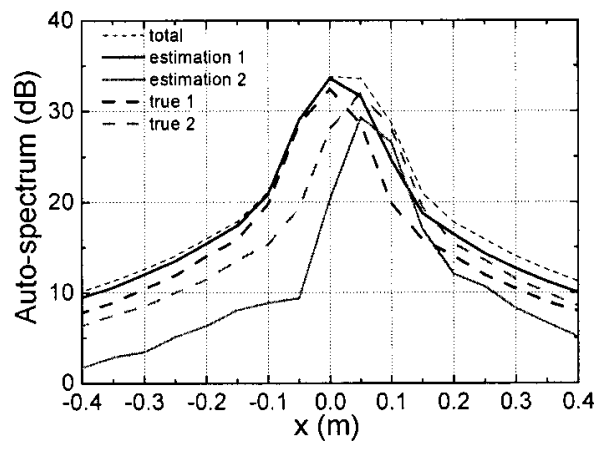

FIG. 18. Decomposition result of two monopoles, $d=5 \mathrm{~cm}$, and the same peak level (total: the autospectrum of pressure on the source plane, estimation 1: the first estimated contribution, estimation 2: the second estimated contribution, true 1: the true contribution of the first source, true 2 : the true contribution of the second source).

\section{APPENDIX C: SOME EXAMPES OF DECOMPOSITION ERROR}

In this appendix we illustrate some numerical examples of decomposition errors. Spatial overlap generates a decomposition error. However, it is difficult to know how much is the spatial overlap or decomposition error. That is because the spatial overlap is directly related to the spatial distributions of individual sources, which is the objective of the decomposition. Therefore some numerical examples of this appendix cannot provide perfect guidelines on the spatial overlap. However, the examples will be helpful for the judgment of decomposition results because they have some common symptoms and features.

In this appendix we focuse on the behavior at peak points because the contribution of one source is more distorted at the peak points of other sources. Let us consider a two-input/single-output system (Sec. II B). Let $\hat{s}_{y 1 y 1}$ and $\hat{s}_{y 2 y 2}$ be the estimators of the true $s_{y 1 y 1}$ and $s_{y 2 y 2}$ [Eq. (4)]. If the $\hat{s}_{y 1 y 1}$ is $s_{y 1 y 1}+\epsilon$, then the $\hat{s}_{y 2 y 2}$ is $s_{y 1 y 1}-\epsilon$ because their sum is equal to the total output spectrum. That is, the two estimators have the same absolute errors. If $\hat{s}_{y 1 y 1} \ll \hat{s}_{y 2 y 2}$, however, the $\hat{s}_{y 1 y 1}$ has a large relative error and the $\hat{s}_{y 2 y 2}$ has a small relative error. Therefore the contribution of one source has a large error at the peak points of other sources.

Figure 17 shows the specifications of the numerical simulations on two incoherent sources. The hologram $\left(z_{H}\right)$ and source planes $\left(z_{S}\right)$ were located at $10 \mathrm{~cm}$ and 0 . The measurement spacing was $5 \mathrm{~cm}$ and the number of measurement points was $32 \times 32$. The frequency was $343 \mathrm{~Hz}$. Zeros were added so that the number of data including the zeros was $64 \times 64$. The spatial window minimizing errors ${ }^{17}$ and the wave number filter proposed by Veronesi and Maynard ${ }^{18}$ were used. For the decomposition, this simulation used the maximum pressure on the plane located at $z_{D}=-5 \mathrm{~cm}$ instead of the source plane. The plane at the back of the source plane is often more useful than the source plane. ${ }^{1}$ The variables are the source type (monopole or dipole), the distance $(d=5,10,15 \mathrm{~cm})$ between the sources, and the source strength (or peak level difference: $6,0,-6 \mathrm{~dB}$ ).

Figure 18 is the result of two monopoles, $d=5 \mathrm{~cm}$, and the same peak level. The symptom is the steep decrease of the second contribution near the peak point of the first con- 


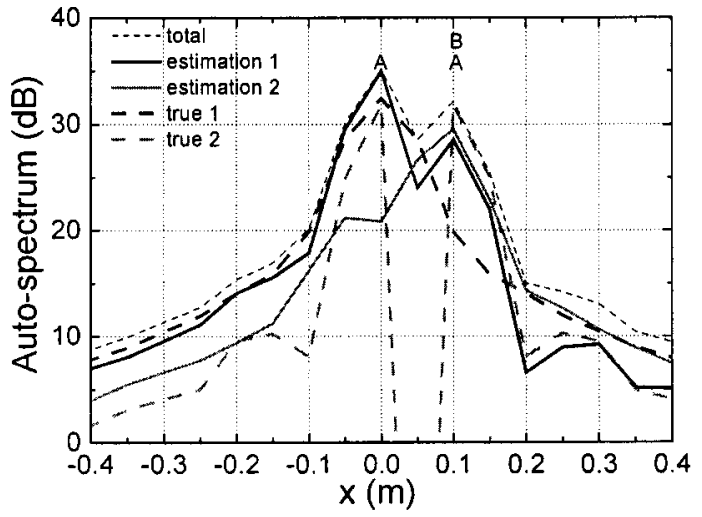

(a) $\mathrm{d}=5 \mathrm{~cm}$, the same peak level

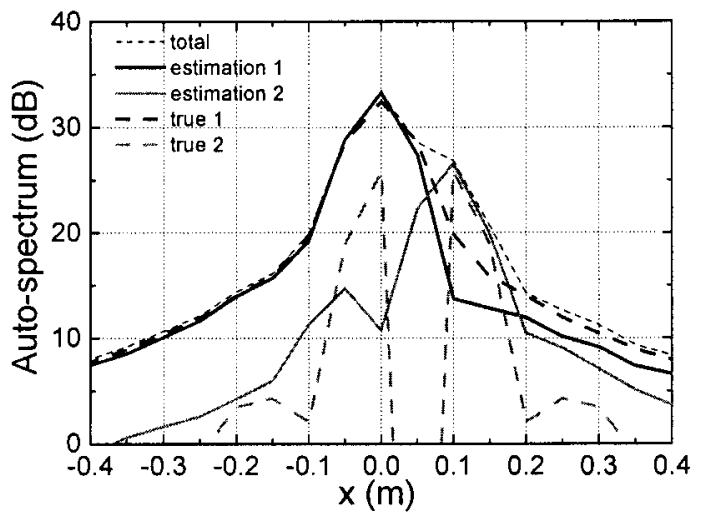

(b) $\mathrm{d}=5 \mathrm{~cm}$, dipole peak $=$ monopole peak $-6 \mathrm{~dB}$

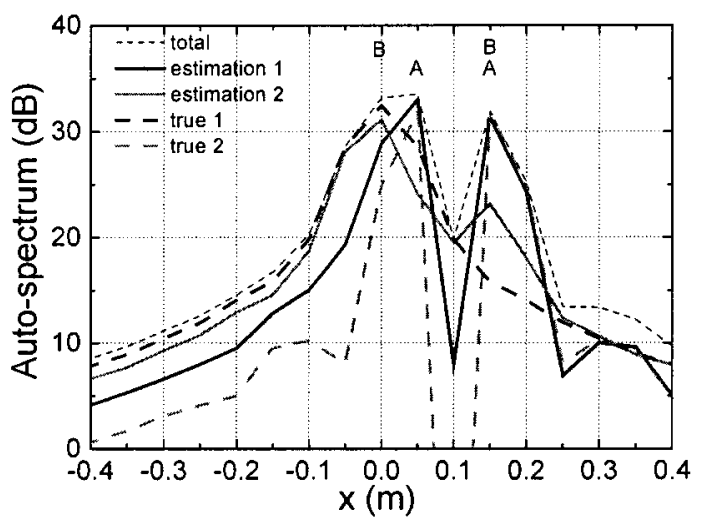

(c) $\mathrm{d}=10 \mathrm{~cm}$, the same peak level

FIG. 19. Decomposition results of a monopole and a dipole (total: the autospectrum of pressure on the source plane, estimation 1: the first estimated contribution, estimation 2: the second estimated contribution, true 1: the true contribution of the first source, true 2: the true contribution of the second source, A and B: the peak points of the first and second contributions).

tribution. The first and second are over- and underestimated, respectively. When there are two monopoles, the results of other distances and peak levels showed the same phenomena as Fig. 18, or were well decomposed.

Figure 19 shows the results with a monopole and a dipole. Figure 19(a) is the result of $d=5 \mathrm{~cm}$ and the same peak level. The symptom is that the peak point (B) of the second is also the peak point (A) of the first. All the contributions were largely distorted. Figure 19(b) shows the result when the dipole peak is $6 \mathrm{~dB}$ less than the monopole peak and $d$ $=5 \mathrm{~cm}$. The symptom is the steep decrease of the first near

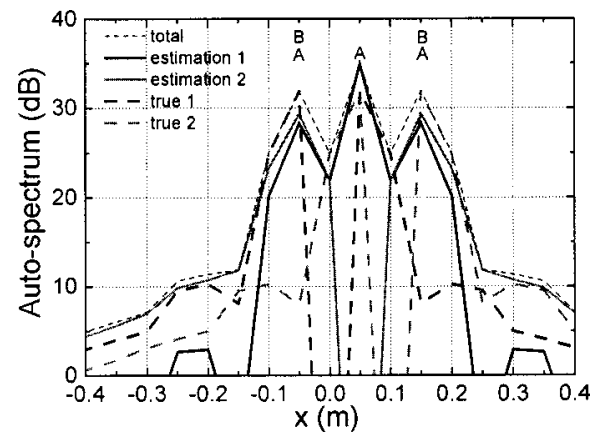

(a) $\mathrm{d}=10 \mathrm{~cm}$, the same peak level

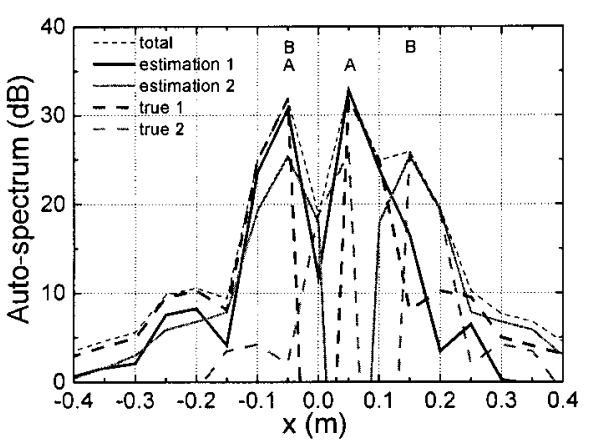

(b) $\mathrm{d}=10 \mathrm{~cm}$, peak difference $=6 \mathrm{~dB}$

FIG. 20. Decomposition results of two dipoles (total: the auto-spectrum of pressure on the source plane, estimation 1: the first estimated contribution, estimation 2: the second estimated contribution, true 1: the true contribution of the first source, true 2: the true contribution of the second source, A and B: the peak points of the first and second contributions).

the peak point of the second. Only the second contribution has a large error. Figure $19(\mathrm{c})$ is the result of $d=10 \mathrm{~cm}$ and the same peak level. One of the peak points (B) of the second is also the peak point (A) of the first, but the other is not. The second is largely distorted only near the coinciding peak point. The results of other distances and peak levels were similar to those in Fig. 19, or were well decomposed.

Figure 20 shows the results when there are two dipoles. Fig. 20(a) is the result of $d=10 \mathrm{~cm}$ and the same peak level. All the peak points (B) of the second are also those of the first. All the contributions had large errors. This is the same phenomenon as Fig. 3(a). Figure 20(b) is the result when the peak level difference is $6 \mathrm{~dB}$, and is the same as Fig. 19(c). The results of other distances and peak levels were the same as in Fig. 20, or well decomposed.

From the above observation, Table I summarizes the common symptoms and features of the decomposition errors.

\section{APPENDIX D: CONSIDERATION FOR A STEP-BY-STEP SCANNING METHOD}

A measurement problem always includes "how to measure" and "how to check the accuracy." The "how to measure" of the step-by-step scanning method ${ }^{4,5}$ is related to the number and positions of reference microphones. The "how to check the accuracy" is related to the evaluation method of the error in the real measurement.

It is well known that the number of reference microphones has to be more than that of incoherent sources. ${ }^{4}$ This implies that one has to use as many reference microphones as 
TABLE I. Common symptoms and features of decomposition error.

\begin{tabular}{lll}
\hline \hline Symptom & \multicolumn{1}{c}{ Feature } & Example \\
\hline $\begin{array}{l}\text { The second contribution steeply } \\
\text { decreases near the peak point of } \\
\text { the first contribution. }\end{array}$ & $\begin{array}{l}\text { The first contribution is over- } \\
\text { estimated and the second } \\
\text { contribution is underestimated. } \\
\text { The first contribution steeply } \\
\text { decreases near the peak point of } \\
\text { the second contribution. }\end{array}$ & $\begin{array}{l}\text { a large error. } \\
\begin{array}{l}\text { All the peak points of the second } \\
\text { contribution coincide with those of } \\
\text { the first contribution. }\end{array}\end{array}$ \\
$\begin{array}{l}\text { Some peak points of the second } \\
\text { contribution coincide with those of } \\
\text { the first contribution. }\end{array}$ & $\begin{array}{l}\text { All the contributions have large } \\
\text { errors. }\end{array}$ & Figs. 19(a), 20(a) \\
\hline \hline
\end{tabular}

possible if the number of sources is unknown. From this viewpoint, the six-speaker and vortex shedding experiments used as many reference microphones as possible. This strategy can be inefficient if the number of sources is small, but does not decrease the accuracy. The singular value decomposition of the spectral matrix may be useful for identifying the number of sources. ${ }^{19}$

If there are enough reference microphones, the step-bystep scanning method does not depend on their positions. Therefore the reference microphones can be farfield like the six-speaker and vortex shedding experiments if the signal-tonoise ratio is high enough. At times, it has been proposed that reference microphones be placed near the sources. That is because the reference microphones are often used for the partial field decomposition as well as the construction of the hologram spectral matrix. If the objective of the step-by-step

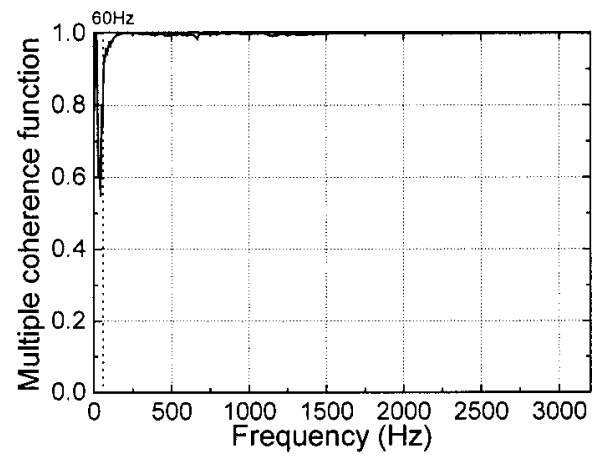

(a) 6-speaker experiment

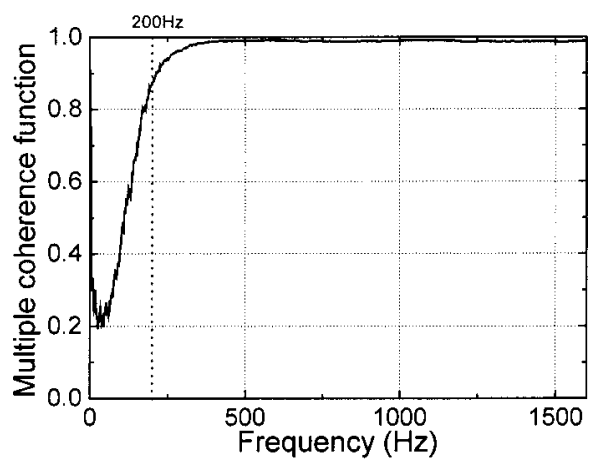

(b) vortex shedding experiment

FIG. 21. Spatially averaged multiple coherence functions of the main text experiments. scanning is only the construction of the hologram spectral matrix, it is not necessary to place the reference microphones near the sources.

For the error evaluation, let us define an error derived from a matrix norm ${ }^{16}$ as

$$
\boldsymbol{\epsilon}_{H H}=\sum_{m=1}^{N} \sum_{n=1}^{N}\left|s_{H m H n}-\hat{s}_{H m H n}\right| / \sum_{m=1}^{N} \sum_{n=1}^{N}\left|s_{H m H n}\right|,
$$

where the $s_{H m H n}$ and $\hat{s}_{H m H n}$ are the $(m, n)$ th element of the true and measured hologram spectral matrix $\left(S_{H H}\right.$ and $\left.\hat{S}_{H H}\right)$. However, all the elements of the true cannot be obtained. As an alternative, let us define a modified error as

$$
\epsilon_{H H}^{\prime}=\sum_{n=1}^{N}\left|s_{H n H n}-\hat{s}_{H n H n}\right| / \sum_{n=1}^{N} s_{H n H n} .
$$

The modified error can be calculated when there is no noise because the true autospectra can be measured by step-by-step scanning. Because the $\hat{s}_{H n H n}$ is the coherent output spectrum of $s_{H n H n}$ with respect to the reference signals, the $\hat{s}_{\mathrm{HnHn}}$ is always less than the $s_{H n H n} .{ }^{13}$ Therefore Eq. (D2) can be rewritten as

$$
\epsilon_{H H}^{\prime}=1-\gamma_{H: R}^{2},
$$

where

$$
\epsilon_{H H}^{\prime}=\sum_{n=1}^{N} \hat{s}_{H n H n} / \sum_{n=1}^{N} s_{H n H n} .
$$

As the $\gamma_{H: R}^{2}$ approaches 1, the error becomes smaller. We call the $\gamma_{H: R}^{2}$ a spatially averaged multiple coherence function. It is the expansion of a multiple coherence function. ${ }^{11,13}$ Figure 21 shows its values in the speaker and vortex shedding experiments. If the criterion is 0.8 (about $1 \mathrm{~dB}$ error), the speaker and vortex shedding experiments are reliable at frequencies higher than 60 and $200 \mathrm{~Hz}$, respectively.

${ }^{1}$ K.-U. Nam and Y.-H. Kim, "Visualization of multiple incoherent sources by the backward prediction of near-field acoustic holography," J. Acoust. Soc. Am. 109, 1808-1816 (2001).

${ }^{2}$ E. G. Williams, J. D. Maynard, and E. Skudrzyk, "Sound source reconstruction using a microphone array," J. Acoust. Soc. Am. 68, 340-344 (1980)

${ }^{3}$ E. G. Williams and J. D. Maynard, "Holographic imaging without the wavelength resolution limit," Phys. Rev. Lett. 45, 554-557 (1980).

${ }^{4}$ J. Hald, "STSF-A unique technique for scan-based near-field acoustic 
holography without restrictions on coherence," B\&K Technical Review No. 1, 1989.

${ }^{5}$ K. B. Ginn and J. Hald, "STSF-Practical instrumentation and applications," B\&K Technical Review No. 2, 1989.

${ }^{6}$ D. Hallman and J. S. Bolton, "Multi-reference near-field acoustical holography," in Proceedings of Inter-Noise '92, 1992 International congress on noise control engineering, Held in Toronto, Canada on 1992 July, edited by Gilles A. Daigle and Michael R. Stinson, International Institute of Noise Control Engineering, 1992, pp. 1165-1170.

${ }^{7}$ H.-S. Kwon and J. S. Bolton, "Partial field decomposition in nearfield acoustical holography by the use of singular value decomposition and partial coherence procedures," in Proceedings of Noise-Con '98, the 1998 national conference on noise control engineering, Held in Ypsilanti, Michigan on 1998 April, edited by J. Stuart Bolton and Luc Mongeau, The Institute of Noise Control Engineering of the USA, 1998, pp. 649-654.

${ }^{8}$ R. J. Ruhala and C. B. Burroughs, "Separation of leading edge, trailing edge, and sidewall noise sources from rolling tires," in Proceedings of Noise-Con'98, the 1998 national conference on noise control engineering, Held in Ypsilanti, Michigan on 1998 April, edited by J. Stuart Bolton and Luc Mongeau, The Institute of Noise Control Engineering of the USA, 1998, pp. 109-114.

${ }^{9}$ M. A. Tomlinson, "Partial source discrimination in near field acoustic holography,” Appl. Acoust. 57, 243-261 (1999).

${ }^{10}$ S. M. Price and R. J. Bernhard, "Virtual coherence: A digital signal processing techniques for incoherent source identification," in Proceedings of
IMAC IV, the 4th International Modal Analysis Conference, Held in Los Angeles, California on 1986, February, Society for experimental mechanics, 1986 , pp. 1256-1262.

${ }^{11}$ G. M. Jenkins and D. G. Watts, Spectral Analysis and its Applications (Holden-Day, San Francisco, 1968), pp. 458-513.

${ }^{12}$ C. J. Dodds and J. D. Robson, "Partial coherence in multivariate random process," J. Sound Vib. 42, 243-249 (1975).

${ }^{13}$ J. S. Bendat and A. G. Piersol, Random Data: Analysis and Measurement Procedures, 2nd ed. (Wiley, New York, 1986), pp. 164-251.

${ }^{14}$ H.-S. Kwon and Y.-H. Kim, "Moving frame technique for planar acoustic holography,” J. Acoust. Soc. Am. 103, 1734-1741 (1998).

${ }^{15}$ S.-H. Park and Y.-H. Kim, "An improved moving frame acoustic holography for coherent band-limited noise," J. Acoust. Soc. Am. 104, 31793189 (1998).

${ }^{16} \mathrm{P}$. Lancaster and M. Tismenetsky, The Theory of Matrices with Application, 2nd ed. (Academic, New York, 1985), pp. 71-440.

${ }^{17}$ H.-S. Kwon and Y.-H. Kim, "Minimization of bias error due to windows in planar acoustic holography using a minimum error window," J. Acoust. Soc. Am. 98, 2104-2111 (1995).

${ }^{18}$ W. A. Veronesi and J. D. Maynard, "Nearfield acoustic holography (NAH): 2 Holographic reconstruction algorithms and computer implementation," J. Acoust. Soc. Am. 81, 1307-1322 (1987).

${ }^{19}$ M. S. Kompella, P. Davies, R. J. Bernhard, and D. A. Ufford, "A technique to determine the number of incoherent sources contributing to the response of a system," Mech. Syst. Signal Process. 8, 363-380 (1994). 